\title{
A review and content analysis of engagement, functionality, aesthetics, information quality, and change techniques in the most popular commercial apps for weight management
}

\author{
Marco Bardus ${ }^{1,2^{*}}$ D, Samantha B. van Beurden ${ }^{2}$, Jane R. Smith ${ }^{2}$ and Charles Abraham²
}

\begin{abstract}
Background: There are thousands of apps promoting dietary improvement, increased physical activity (PA) and weight management. Despite a growing number of reviews in this area, popular apps have not been comprehensively analysed in terms of features related to engagement, functionality, aesthetics, information quality, and content, including the types of change techniques employed.

Methods: The databases containing information about all Health and Fitness apps on GP and iTunes (7,954 and 25,491 apps) were downloaded in April 2015. Database filters were applied to select the most popular apps available in both stores. Two researchers screened the descriptions selecting only weight management apps. Features, app quality and content were independently assessed using the Mobile App Rating Scale (MARS) and previously-defined categories of techniques relevant to behaviour change. Inter-coder reliabilities were calculated, and correlations between features explored.

Results: Of the 23 popular apps included in the review 16 were free (70 \%), 15 (65\%) addressed weight control, diet and PA combined; 19 (83 \%) allowed behavioural tracking. On 5-point MARS scales, apps were of average quality ( $M d=3.2, I Q R=1.4)$; "functionality" ( $M d=4.0, I Q R=1.1)$ was the highest and "information quality" (Md = 2.0, $\mathrm{IQR}=1.1$ ) was the lowest domain. On average, 10 techniques were identified per app (range: 1-17) and of the 34 categories applied, goal setting and self-monitoring techniques were most frequently identified. App quality was positively correlated with number of techniques included ( $r h o=.58, p<.01$ ) and number of "technical" features $(r h o=.48, p<.05)$, which was also associated with the number of techniques included (rho $=.61, p<.01)$. Apps that provided tracking used significantly more techniques than those that did not. Apps with automated tracking scored significantly higher in engagement, aesthetics, and overall MARS scores. Those that used change techniques previously associated with effectiveness (i.e., goal setting, self-monitoring and feedback) also had better "information quality".

(Continued on next page)
\end{abstract}

\footnotetext{
* Correspondence: marco.bardus@gmail.com

${ }^{1}$ Department of Health Promotion and Community Health, American

University of Beirut, Riad El Solh, Beirut 1107 2020, Lebanon

${ }^{2}$ Psychology Applied to Health group, University of Exeter Medical School, St

Luke's Campus, Heavitree Road, Exeter EX1 2LU, United Kingdom
} 
(Continued from previous page)

Conclusions: Popular apps assessed have overall moderate quality and include behavioural tracking features and a range of change techniques associated with behaviour change. These apps may influence behaviour, although more attention to information quality and evidence-based content are warranted to improve their quality.

Keywords: Smartphone, Mobile apps, Mobile health (mhealth), Behaviour change techniques, Weight loss, Weight management

\section{Background}

There are thousands of apps available in the health and fitness categories of iTunes and Google Play (GP). The most popular apps are used for tracking physical activity (PA) (38\%), diet (31\%), and managing weight (12\%). With so many apps available, it has become challenging to identify those with most potential to support weight loss and reduce obesity $[1,2]$. While users select health apps according to perceived design quality and ease of use [3], researchers have so far evaluated apps in terms of more scientific parameters, including adherence to evidence-base and theoretical principles in addition to formal evaluations of design quality. In our recent systematic scoping review of literature on mobile phone and Web 2.0 technologies for weight management [4], we identified 20 reviews and content analyses of apps [5-24]. The majority of these $(14 / 20,70 \%)$ assessed the presence of theoretical components $[5-9,11,13,14,16$, 18, 21-23]; the remainder focused either on evaluating the apps' design and usability qualities [12, 19, 20, 24], or technical functionalities (e.g., behavioural tracking) $[10,17]$. The reviews of theoretical components assessed in particular the presence of constructs derived from behavioural theories $[6,9,22,23]$, tailoring principles [5], evidence-based strategies [7, 16, 18, 21], or techniques designed to promote behaviour change $[8,11,13,14]$, without evaluating functionality and usability. Conversely, the reviews that investigated features related to usability or functionality did not consider theoretical components. Hence, a comprehensive evaluation of the quality of such apps and whether there are relationships between these aspects is lacking.

With regards to behavior change potential, the review evidence specifically analysing behavioural content in weight management apps is limited. The majority of available reviews focus on PA and fitness apps, so it is unclear whether findings from these might extend to broader weight loss apps that do not just focus on PA. Recent reviews demonstrated the importance of incorporating diet in behavioural interventions for weight management, as PA alone has shown only modest effects [25], whereas dietary restriction is an effective weight loss strategy [26]. Additionally, diet combined with PA has beneficial effects on weight maintenance and prevention of weight regain [25-27], which is also aligned with current public health recommendations (e.g., NICE guidelines) for weight management [28]. Indeed, only one review analysed the presence of behavioural components by including both PA and dietary apps [11], but included only apps available on iTunes until November 2012. Even though iTunes App Store covers a large and important part of the apps market, Google Play store recently overtook iTunes by offering a number of apps larger than iTunes [29, 30]. Also, recent industry reports show that Android's market share accounted for more than $81 \%$ in 2014, compared to the $15 \%$ obtained by Apple iOS [31, 32].

A fruitful stream of research has developed a theorybased classification system (taxonomy) that identified and classified various change techniques (also referred as "behaviour change techniques"), designed to influence a variety of psychological processes and mechanisms underpinning behaviour change [33, 34]. Several systematic reviews of weight loss interventions in general have identified various techniques, which were associated with greater effectiveness [35]. These include goal setting, feedback, and self-monitoring [2, 36, 37]. A recent meta-analysis also found larger effects for weight loss interventions incorporating modelling techniques categorised as "demonstration of behaviour" [37]. The four reviews that explicitly assessed technique content in PA and fitness apps $[8,11,13,14]$ identified varying numbers of techniques, ranging from 1 [8] to 18 per app [11]. The most frequently identified technique types were: prompting goal setting, facilitating selfmonitoring, and provision of feedback [11, 13, 14], as well as "demonstration of behaviour", and "prompting or facilitating social support seeking" [8, 11]. Inclusion of defined technique types has only been assessed in PA and fitness apps so it remains unclear whether weight management apps, more generally, employ the same technique types, or whether these are only used in PA apps.

Additionally, the reviews assessing "usability" of apps provide evaluations based on heuristics for general user interfaces developed by Jacob Nielsen almost 20 years ago $[38,39]$, or use inductively-developed approaches $[12,17]$. They provide generally descriptive, qualitative 
evaluations of users' ratings, comments or feedback, and none utilise instruments specifically developed to assess the design and usability of smartphone apps, such as the Mobile App Rating Scale (MARS) [40], which provides app quality ratings for engagement, functionality, aesthetics, and information quality. This instrument has been validated with mental health apps [40] but has not been applied to weight management apps.

To address these gaps, the aim of this study was to evaluate both the quality and content of popular weight management apps available from both iTunes and GP, to answer the following research questions: (1) What is the overall quality of these apps in terms of engagement, functionality, aesthetics, and information quality? (2) What type of change techniques are included in these apps? (3) What are the relationships between user ratings, app quality, other app features, and techniques included, specifically techniques previously found to be associated with weight loss?

\section{Methods}

Sources of information, selection and coding procedure

App selection proceeded in several steps. First, the mHealthApps repository [41], available in the first quarter of 2015 was downloaded and imported into Excel. The repository included details about all apps available in the Health and Fitness category for both iTunes and GP (US stores). Second, a set of database filters was used to include only popular apps, based on the number of downloads and average user ratings. Although app stores do not provide detailed download information, it is possible to estimate this using formulas proposed by Garg and Telang [42], as a function of an app's ranking. On GP, apps were ranked according to average user ratings, weighted by the number of ratings and the 15-level ordinal category 'number of installs' (ranging from $1=1-$ 5 ' to $\left.15={ }^{\prime} 10,000,000-50,000,000 '\right)$. On iTunes, apps were ranked according to average rating weighted by the number of reviews, as the repository does not include information about number of installs.

Third, apps were excluded if they: (a) had an estimated number of downloads below 100, (b) received user ratings below 4 (as done elsewhere $[6,7,9]$ ), (c) were not available on both iTunes and GP and (d) were free apps with limited functionality which is only unlocked by purchasing the full version (i.e., "freemium") [22]. In GP, freemium apps were filtered out as the database contains the variable "in-app purchases"; in iTunes in-app purchases were manually checked.

Fourth, the first author and a collaborator read the descriptions of the apps and applied further inclusion criteria. Apps were included if they addressed "weight management", which consists of both PA and dietary behavioural strategies [28], considering the limited role of
PA and the predominant role of dietary strategies for effective weight loss $[25,27]$, and the importance of the combination of PA and diet for long-term effects on weight [28]. This allowed the exclusion of apps that focused only on PA and fitness. We also excluded apps that focused on other aspects of health (maternal health, mental health, etc.). Apps were excluded if their description was not available in English. The selection process is summarised in Fig. 1.

Lastly, two reviewers downloaded the selected apps and independently tested them using an iPhone $5 \mathrm{~S}$ (iOS 9.0.2) and a Samsung Galaxy S4, GT-19505 (Android 5.0.2). They first familiarised themselves with the app, used it for approximately 2 days, and independently documented features and evaluated the apps using online forms.

\section{Data extraction, evaluation criteria and instruments}

General and technical information were extracted for descriptive purposes. App name, identification number (ID), version, producer, price, average ratings, and total ratings were retained from the databases. General aspects included "behavioural focus" (i.e., weight management, diet or PA). Technical features included the ability to: track behaviour manually or semi-automatically, share on social media; have an app community; protect data with password; limit access by requiring login; have app reminders; function without web access; work in the background. The total number of features was calculated (range: 0-7), as used in the Mobile App Rating Scale study [40].

App quality was evaluated using the MARS scale [40], which includes 19 items grouped in four domains: 1) engagement (entertainment, interest, customisation, interactivity, and target group); 2) functionality (performance, ease of use, navigation, gestural design); 3) aesthetics (layout, graphics, visual appeal); 4) information quality (accuracy of app description, goals, quality and quantity of information, visual information, credibility, evidence base). All items are measured on a 5-point scale $(1=$ inadequate to $5=$ excellent). A score for each domain is computed as the mean of the items in that domain; an overall score is computed as an average across the domains [25].

The presence or absence of techniques used to promote change was assessed. Categories of change techniques were derived from systematic reviews of weight loss interventions $[2,36,37]$ and similar recent reviews of apps for diet and PA $[8,11,13,14]$. Definitions were derived from taxonomies presenting definitions of technique types $[33,34]$ and 34 relevant categories were applied. Definitions were adopted form one taxonomy [32] and were modified, where necessary, to allow meaningful technique categorisation. Relevant categories were 


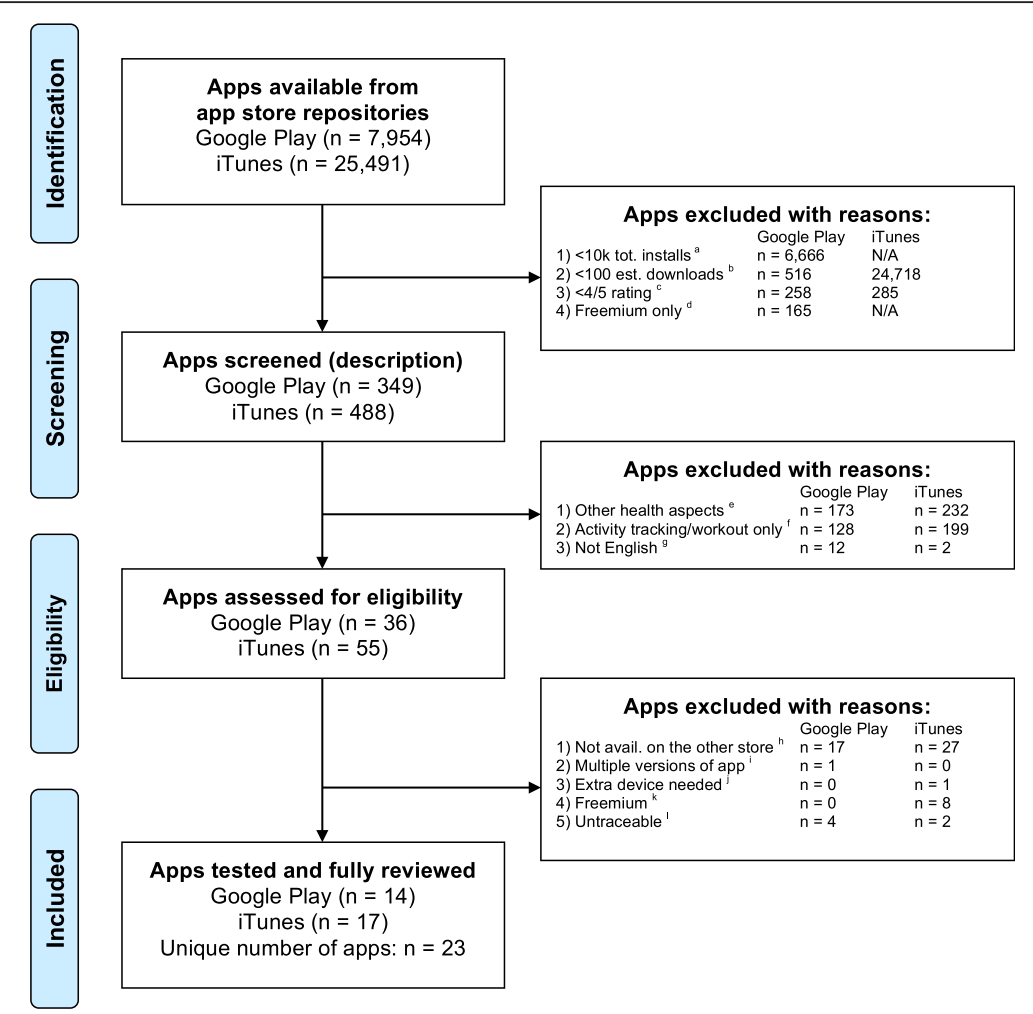

Fig. 1 Flow chart of the selection process for apps included in the review. Legend: a Apps that were downloaded less than 10,000 times. In Google Play, the category 'Installs' includes the information 15 levels ranging from " $1-5$ " to "10,000,000-50,000,000". A popularity index, based on the category of installs, was determined to estimate the number of downloads, as described in Garg and Telang's formula [53]. ${ }^{b}$ Apps that were downloaded less than 100 times a day, based on the rank of the apps. ${ }^{C}$ Apps that received a rating below 4. ${ }^{\mathrm{d}}$ Apps that were classified as having "in-app purchases" (i.e., " freemium"). ${ }^{\text {e }}$ Apps that addressed other health aspects different from weight management or related behaviours (diet and PA), such as smoking, mental health, pregnancy, etc. ${ }^{f}$ Apps that were workout or activity tracking apps without the aim to weight loss. ${ }^{g}$ Apps whose description was not in English. ${ }^{\mathrm{h}}$ Apps that did not have a respective counterpart on the other app store. ${ }^{\mathrm{i}}$ Apps that had more than one version (e.g., HD, lite, pro); the basic, fully-functional version was chosen. ${ }^{j}$ Apps that required an external device (e.g., monitor, wrist band) to function. ${ }^{k}$ Apps that were either free or paid but the paid version did not have additional and fully functional features. ${ }^{~}$ Apps that were not available to download after the selection or that were not available for download on the respective devices iPhone $5 \mathrm{~S}$ (iOS 9.0.2) and Samsung Galaxy S4, GT-19505 (Android 5.0.2)

retained to ensure comprehensive coding of app content and the number of included techniques per app was calculated (Additional file 1: Table S1). Binary variables indicating the presence or absence of technique types found to be associated with effectiveness (i.e., goal setting, self-monitoring, feedback [33, 37, 43]) were included in the analyses.

\section{Interrater reliabilities}

Gwet's AC1 was used to assess agreement in the selection process [44]. Ordinal and nominal Krippendorf's alphas (Kalpha) [45] were used to estimate inter-coder reliability for the MARS scales and change technique categorisation. Reliability estimates below .70 were discussed to check whether differences existed between operative systems or in the actual application of the scales. Disagreements were solved through discussion.
Reliability estimates and correlations are provided in Additional file 1: Tables S1 and Additional file 2: Table S2.

\section{Analyses}

Spearman's correlations were used to explore the relationships among popularity/ratings, total features, MARS scores, and number of techniques. Independent samples t-tests and one-way ANOVAs (or nonparametric alternatives where indicated) were used to test differences among identified app categories. All tests were carried out in $R$ (v. 3.2.2).

\section{Results}

Database filtering and screening results

The GP and iTunes repositories contained 7,954 and 25,491 apps, respectively. Database filtering left 349 
and 488 apps for screening, with 313 apps subsequently excluded from GP (99\% agreement, $\mathrm{AC} 1=.98,95 \%$ $\mathrm{CI}=.97-1.00)$ and 433 apps from iTunes (99\% agreement, $\mathrm{AC} 1=.99,95 \% \mathrm{CI}=.98-1.00)$. The selection process and reasons for exclusion are summarised in Fig. 1. The final sample consisted of 23 unique apps.

\section{General characteristics of the selected apps}

Sample descriptive data are summarised in Table 1, while app characteristics are presented in Additional file 3: Table S3. Most of the apps were free (16/23, $70 \%)$. The average price of paid apps was $\$ 3.49$ on iTunes and $\$ 2.99$ on GP. The median user rating was $4.5(\mathrm{IQR}=.5)$ on iTunes and 4.2 (IQR =.6) on GP. The best-rated apps on iTunes were YouFood and Weilos, while Ultimate Food Value Diary was the best on GP. The median number of ratings was $1,436.5(\mathrm{IQR}=5,186)$ on iTunes and 3,784 $(\mathrm{IQR}=9037)$ on GP. MyFitnessPal received the most ratings on both stores. No differences were detected between paid and free apps with regards to user ratings, number of ratings, app quality, number of techniques, and number of features.

\section{Features of the selected apps}

The Android and iOS versions of the apps presented quite different technical features, as shown by lower

Table 1 Descriptive data for the reviewed apps

\begin{tabular}{|c|c|c|c|c|}
\hline & n (\%) & $\mathrm{M}(\mathrm{SD})$ & $\mathrm{Md}(\mathrm{IQR})$ & Range \\
\hline \multicolumn{5}{|l|}{ App basic descriptive information } \\
\hline Paid apps on iTunes, and price (\$) & $6(26 \%)$ & $3.49(.55)$ & $3.49(1.0)$ & $2.99-3.99$ \\
\hline Paid apps on GP, and price (\$) & $5(22 \%)$ & $3.27(.70)$ & $2.99(1.0)$ & $2.99-3.99$ \\
\hline Avg. user rating on iTunes (scale: 1-5) & $22(96 \%)$ & $4.3(.6)$ & $4.5(.5)$ & $2.0-5.0$ \\
\hline Avg. user rating on GP (scale: 1-5) & $23(100 \%)$ & $4.1(.6)$ & $4.2(.6)$ & $2.4-4.7$ \\
\hline Number of ratings on iTunes (count) & $22(96 \%)$ & $23380.7(92819.4)$ & $1436.5(5186)$ & $11-438279$ \\
\hline Number of ratings on GP (count) & $23(100 \%)$ & $48257.0(174679.8)$ & $3784(9037)$ & $31-836597$ \\
\hline Number of technical features per app (0-7) & $23(100 \%)$ & $4.4(2.2)$ & $5(4)$ & $1-12$ \\
\hline \multicolumn{5}{|l|}{ Presence of technical features } \\
\hline Allows behavioural tracking & $19(83 \%)$ & - & - & - \\
\hline Manual and semi-automated tracking & $9(82 \%)$ & - & - & - \\
\hline Allows sharing & $8(9 \%)$ & - & - & - \\
\hline Has app community & 14 (15\%) & - & - & - \\
\hline Requires login & $13(14 \%)$ & - & - & - \\
\hline Password & 10 (11\%) & - & - & - \\
\hline Works in background & $14(15 \%)$ & - & - & - \\
\hline Notifications & $18(20 \%)$ & - & - & - \\
\hline Needs internet to work & $15(16 \%)$ & - & - & - \\
\hline \multicolumn{5}{|l|}{ MARS app quality ratings (1-5) } \\
\hline Engagement & $23(100 \%)$ & $3.0(.9)$ & $2.8(1.2)$ & $1.3-5.0$ \\
\hline Functionality & $23(100 \%)$ & $3.8(.9)$ & $4.0(1.1)$ & $1.8-5.0$ \\
\hline Aesthetics & $23(100 \%)$ & $3.4(1.2)$ & $3.8(2.7)$ & $1.5-4.8$ \\
\hline Information quality & $23(100 \%)$ & $2.2(.7)$ & $2.0(1.1)$ & $1.2-4.1$ \\
\hline Total score & $23(100 \%)$ & $3.1(.8)$ & $3.2(1.4)$ & $1.9-4.6$ \\
\hline Number of change techniques & $23(100 \%)$ & $9.3(4.0)$ & $10.0(6.0)$ & $1.0-17.0$ \\
\hline \multicolumn{5}{|l|}{ Presence of effective techniques } \\
\hline Allows goal setting (GS) only & $1(4 \%)$ & - & - & - \\
\hline GS and self-monitoring (SM) & $2(9 \%)$ & - & - & - \\
\hline GS, SM and feedback (F) & $16(70 \%)$ & - & - & - \\
\hline GS, SM, F and description of behaviour (DB) & $2(9 \%)$ & - & - & - \\
\hline SM only & $1(4 \%)$ & - & - & - \\
\hline $\mathrm{SM}$ and $\mathrm{F}$ & 1 (4 \%) & - & - & - \\
\hline
\end{tabular}


reliability estimates (Md Kalpha $=.50, \mathrm{IQR}=.55$ ). Only the features that were identified by both reviewers were counted. The median number of features offered by the apps was 5 out 7, ranging from 0 (Fast Food Nutrition \& Weight Loss) to 7 (NexTrack). Most apps offered notifications $(18 / 23,82 \%)$, needed web access to function (15 apps, $68 \%$ ), worked in background, and had a community (14 apps, $64 \%$ ).

Nineteen apps (83 \%) allowed "behavioural tracking"; the remaining four were food information apps (Fast Food Nutrition \& Weight Loss and Foods That Burn Fat), and weight-loss communities (Weilos and YouFood). Among behavioural tracking apps, 13 (68 \%) allowed tracking of weight, diet, and PA, while the remaining six allowed tracking of only one or two of these. Behavioural tracking was either manual (10 apps, $53 \%$ ) or semiautomated (9 apps, $47 \%$ ). Examples of semi-automated logging included: syncing weight information from digital scales such as Withings (e.g., Lark, MyDietDiary); gathering activity information through built-in motion sensors and GPS (e.g., Lark, MyFitnessPal), or thirdparty devices such as FitBit; food information through barcode scanners (e.g., MyPlate, CalorieCount), speech recognition (Calorie Counter), or natural language processing (Lark).

\section{App quality}

Reliability estimates for quality were good (Md Kalpha $=.80$, $\mathrm{IQR}=.14)$. The average MARS score was 3.1 out of 5 $(\mathrm{IQR}=1.4)$, ranging from 1.9 (Diet Plan and Diet Watchers Diary) to 4.9 (My Diet Coach PRO). "Functionality" was the highest-scoring domain $(\mathrm{Md}=4.0, \mathrm{IQR}=1.1)$, followed by "aesthetics" ( $\mathrm{Md}=3.8, \mathrm{IQR}=2.7)$, "engagement" ( $M d=2.8, I Q R=1.2)$, and "information quality" $(\mathrm{Md}=2.0, \mathrm{IQR}=1.1)$. All domains were significantly and positively associated with one another, except for information quality with functionality (see Table 2).

\section{Change technique categories}

Reliability estimates for technique coding were good (Md Kalpha $=.84, \mathrm{IQR}=.20)$. The average number of techniques per app was $10(\mathrm{IQR}=6)$, ranging from 1 (Fast Food Nutrition \& Weight Loss) to 17 (My Diet Coach PRO). At least one instance was identified for 24 of the 33 technique categories applied. The most frequently identified technique types were: "self-monitoring" (of behaviour: 20 apps, $87 \%$; of outcome: 19 apps, $83 \%$ ), "goal setting" (of outcome: 19 apps, $83 \%$; of behaviour: 13 apps, 57 \%), and "feedback" (of outcomes: 17 apps, $74 \%$; of behaviour 16 apps, $70 \%$ ). Lark and $M y$ Diet Coach PRO, which were based on interactive coaching, included a higher number of techniques than other apps. Technique categories and frequency of identified instances are presented in Additional file 1: Table S1.

\section{Relationship between app quality, features and technique inclusion}

Number of included techniques was positively associated with overall MARS score $(r h o=.57, p<.01)$, engagement (rho $=.49, p<.05)$, functionality ( $r h o=.48, p<.05)$, aesthetics $(r h o=.55, p<.01)$, and number of ratings on iTunes $(r h o=.51, p<.05)$. Number of features was associated with number of ratings on GP $(r h o=.51, p<.05)$ and on iTunes (rho $=.63, p<.01)$, MARS score (rho $=.48, p<.05)$, engagement $(r h o=.64, p<.01)$, aesthetics $(r h o=.52, p<.05)$, and number of techniques $(r h o=.61, p<.01)$. Other correlations were non-significant (see Table 2).

Some general and technical features were related to significant differences in MARS scores and number of techniques. Apps that provided semi-automated tracking included a significantly higher number of techniques $(\mathrm{t}=2.93, \mathrm{df}=21, p<.01,95 \% \mathrm{CI}=1.59$ to 9.43$)$, and had significantly higher MARS scores $(\mathrm{t}=2.20, \mathrm{df}=17, p=.04$, $95 \% \mathrm{CI}=1.49$ to .03$)$, engagement $(\mathrm{t}=2.14, \mathrm{df}=17, p=.05$, $95 \% \mathrm{CI}=1.55$ to .01$)$, and aesthetics $(\mathrm{U}=16.5, \mathrm{z}=-2.34$,

Table 2 Correlations among app ratings, MARS, number of techniques and total number of features

\begin{tabular}{|c|c|c|c|c|c|c|c|c|c|c|}
\hline Variables & 1 & 2 & 3 & 4 & 5 & 6 & 7 & 8 & 9 & 10 \\
\hline 1. Avg. rating iTunes & 1.00 & & & & & & & & & \\
\hline 2. Avg. rating GP & .16 & 1.00 & & & & & & & & \\
\hline 3. User ratings iTunes & .27 & -.05 & 1.00 & & & & & & & \\
\hline 4. User ratings GP & -.02 & .40 & .22 & 1.00 & & & & & & \\
\hline 5. MARS engagement & $.47^{*}$ & .24 & .33 & .25 & 1.00 & & & & & \\
\hline 6. MARS functionality & -.01 & -.01 & .08 & .25 & $.62^{* *}$ & 1.00 & & & & \\
\hline 7. MARS aesthetics & .31 & .26 & .21 & .29 & $.81^{* *}$ & $.80^{* *}$ & 1.00 & & & \\
\hline 8. MARS information quality & -.12 & .29 & -.06 & .22 & .28 & $.54^{* *}$ & $.50^{*}$ & 1.00 & & \\
\hline 9. MARS total score & .24 & .18 & .21 & .26 & $.82^{* *}$ & $.90^{* *}$ & $.96^{* *}$ & $.58^{* *}$ & 1.00 & \\
\hline 10. Number of change techiques & .06 & .00 & $.51^{*}$ & .18 & $.49^{*}$ & $.48^{*}$ & $.55^{* *}$ & $.47^{*}$ & $.58^{* *}$ & 1.00 \\
\hline 11. Number of features & .19 & .07 & $.63^{* *}$ & $.51^{*}$ & $.64^{* *}$ & .33 & $.52^{*}$ & .07 & $.48^{*}$ & $.61^{* *}$ \\
\hline
\end{tabular}

Notes: GP google play, MARS mobile app rating scale. ${ }^{* *} p<.01 ;{ }^{*} p<.05$ 
$p=.02)$. Apps that allowed sharing on social media scored higher on overall MARS score $(\mathrm{t}=2.14, \mathrm{df}=21, p=.04$, $95 \% \mathrm{CI}=.02$ to 1.35$)$, engagement $(\mathrm{t}=3.82, \mathrm{df}=21, p=.02$, $95 \% \mathrm{CI}=.54$ to 1.83$)$, and aesthetics $(\mathrm{U}=26.0, \mathrm{z}=-2.02$, $p=.03)$. Apps that had a community also scored higher on MARS score $(\mathrm{t}=2.29, \mathrm{df}=21, p=.03,95 \% \mathrm{CI} .07$ to 1.35), engagement $(\mathrm{t}=2.56, \mathrm{df}=21, p<.01,95 \% \mathrm{CI} .16$ to 1.59), aesthetics $(\mathrm{U}=25.5, \mathrm{z}=-2.37, p=.02)$, and number of techniques $(\mathrm{t}=3.16, \mathrm{df}=21, p<.01,95 \%$ CI 1.54 to 7.49). Apps that used notifications (i.e., prompts and reminders) scored significantly higher in engagement $(\mathrm{t}=2.55, \mathrm{df}=21, p=.02,95 \% \mathrm{CI} .19$ to 1.88$)$, and number of techniques $(\mathrm{t}=3.07, \mathrm{df}=21, p<.01,95 \%$ CI 1.70 to 8.73). Number of techniques was also higher among apps that required Internet connection to work $(\mathrm{t}=2.84, \mathrm{df}=21, p<.01,95 \% \mathrm{CI} 1.15$ to 7.44). Apps that used the most effective techniques combined scored significantly higher on "information quality" ( $\mathrm{U}=14.00, \mathrm{z}=-2.31, p=.02)$. No other differences were identified.

\section{Discussion}

\section{Principal results}

In this paper we described the features of popular weight management apps, analysing their quality and change technique content. App quality was measured through the MARS scale [40], assessing engagement, functionality, aesthetics, and information quality, to provide a more complete evaluation than use of app store user ratings. It is important to note that some apps, although available on both iTunes and GP, offer slightly different features on each operative system. The popular apps examined were overall of moderate quality, but scored higher in terms of functionality and aesthetics. Even though these domains were not significantly associated with user ratings on GP or iTunes, this confirms the idea that people tend to choose well-designed apps that are functional and easy to use [3]. Apps that provided additional interactive features, such as behavioural tracking and semi-automated options, scored significantly higher in engagement, aesthetics and overall app quality, and were also more highly rated on GP. This suggests that reducing the burden associated with manually logging diet or PA may increase engagement, the appeal of an app, and the chances of its repeated use, hence generating a virtuous circle for the app itself.

Aesthetics and functionality should not be the only aspects considered when designing an app [46]. Information quality achieved the lowest score across the MARS domains, indicating an overall lack of evidence-based content, which is consistent with similar reviews $[6,7,9$, $16,18,21,23]$. This suggests that developers should invest more in evidence-based, data-driven content, which might improve the overall app quality, regardless of the perceived aesthetic and engagement qualities of the app.

Even though apps vary considerably in the number of techniques included, goal setting, self-monitoring and provision of feedback were the most frequently identified types of change techniques. This is consistent with the literature on weight management interventions generally [43] and similar reviews on apps for PA $[8,13,14]$ and PA and diet [11]. From a psychological perspective, then, these apps generally target change mechanisms specified by Control Theory [47]. By contrast, building of behavioural skills was much less evident. For example, "demonstration of behaviour", which has previously been shown to be associated with positive effects on weight [18], was observed in only 2 of 23 apps. These two apps provided libraries and examples of workouts, commonly found in PA apps [11], but not in general weighttracking and food logging apps.

This review linked app quality with the presence of types of change techniques and with features of the apps themselves. Using semi-automated tracking (i.e., simplified self-monitoring), having a community, sharing on social media (i.e., offering social support), and using notifications (i.e., behavioural prompts/cues) were features that were associated with higher app quality. This shows that offering features to support specific techniques might improve the perceived functionality, aesthetics and engagement of the app and lead to repeated use. The app quality indicated by MARS scores was positively correlated with number of techniques included, except for information quality, which was only significantly higher only in apps including techniques previously associated with effectiveness. This suggests that perceived information quality is associated with a specific combination of techniques (i.e., goal setting, feedback, and self-monitoring together), which are commonly associated with effectiveness in behavioural interventions. Generally, higher quality apps use a greater range of techniques. This further reinforces the need for developers to incorporate high quality evidence-based content, including specific techniques targeting specified change mechanisms, to improve the overall quality of the app. Future studies should then investigate whether and how the combined use of a variety of techniques found to be effective in other types of weight loss interventions translate into sustained behaviour change supported by an app.

\section{Strengths and limitations}

This review provided a comprehensive evaluation of app quality, described technical features and identified specific techniques in 23 popular weight management apps. For the first time, this review identified positive correlations among app quality dimensions, number of 
techniques included and app features, which might be useful for users, developers and health care professionals. However, some limitations need to be acknowledged. This review focused explicitly on popular apps, i.e., highly downloaded and rated, and available on both Google Play and iTunes stores to improve comparability among apps currently available on the market. Despite being systematically selected, it remains unclear whether the same features might be found in less popular apps. For feasibility purposes, apps were selected applying thresholds to database filters (e.g., number of downloads, in-app purchases) and a relatively small sample of apps was included. Automatic filtering might have excluded good quality apps that have been misclassified or whose ratings did not reach the thresholds. Apps were evaluated over two days and some features and techniques may have been overlooked as some apps presented new content after repeated use. In addition, considering the dynamic development of apps, app ratings and popularity change over time very quickly. We believe that by focusing on the most popular apps, our conclusions are relatively enduring.

\section{Conclusions}

The popular weight management apps analysed were of moderate quality and provided behavioural tracking features combined with change techniques commonly associated with behaviour change. In addition to functionality and aesthetics, app developers should invest in providing content and employing change techniques known to be effective in changing relevant behaviour patterns in order to improve the user experience and foster behaviour change. Despite growing use of apps in research, additional experimental evaluations of such apps are needed to understand whether the presence of particular content, types of change techniques, is associated with behaviour change.

\section{Additional files}

Additional file 1: Table S1. Number and type of change techniques used and reliability estimates. (PDF $158 \mathrm{~kb}$ )

Additional file 2: Table S2. MARS coding, reliability estimates and correlations. (PDF $179 \mathrm{~kb}$ )

Additional file 3: Table S3. Characteristics and features of the reviewed apps. (PDF $121 \mathrm{~kb}$ )

\section{Abbreviations}

GP: google play; IQR: interquartile range; MARS: mobile app rating scale; Md: median; PA: physical activity.

\section{Competing interests}

The authors declared that they have no competing interests.

\section{Authors' contributions}

MB: Conducted the review, performed the analyses, drafted the manuscript and incorporated all feedback. SVB: Conducted the review, provided intellectual input to the review and manuscript and approved the final version. JS: Provided intellectual input, provided feedback on the manuscript and approved the final version of the manuscript. CA: Provided intellectual input to the design and execution of the review and to the manuscript, provided feedback and approved the final version of the manuscript. All authors read and approved the final manuscript.

\section{Acknowledgements}

The authors wish to thank Ms Laya Samaha, who helped with the screening of the apps. The research was partially supported by the Swiss National Science Foundation (SNSF), through a grant awarded to the first author (ref: P2TIP1_152290), and by the UK National Institute for Health Research (NIHR) Collaboration for Leadership in Applied Health Research and Care of the South West Peninsula (PenCLAHRC). The views expressed in this paper are those of the authors and not necessarily those of SNSF, NIHR or the UK Department of Health.

Received: 3 November 2015 Accepted: 4 March 2016

Published online: 10 March 2016

\section{References}

1. Aguilar-Martinez A, Sole-Sedeno JM, Mancebo-Moreno G, Medina FX, Carreras-Collado R, Saigi-Rubio F. Use of mobile phones as a tool for weight loss: a systematic review. J Telemed Telecare. 2014;20:339-49.

2. Lyzwinski LN. A systematic review and meta-analysis of mobile devices and weight loss with an intervention content analysis. J Pers Med. 2014;4:311-85.

3. Armstrong S. Which app should i use? BMJ. 2015;351:h4597.

4. Bardus M, Smith J, Samaha L, Abraham C. Mobile phone and web 2.0 technologies for weight management: A systematic scoping review. J Med Internet Res. 2015;17:e259.

5. Akker $\mathrm{H}$, Jones VM, Hermens $\mathrm{HJ}$. Tailoring real-time physical activity coaching systems: A literature survey and model. User Model User-Adapt Interact. 2014;24:351-92.

6. Azar KMJ, Lesser LI, Laing BY, Stephens J, Aurora MS, Burke LE, Palaniappan LP. Mobile applications for weight management: Theory-based content analysis. Am J Prev Med. 2013;45:583-9.

7. Breton ER, Fuemmeler BF, Abroms LC. Weight loss-there is an app for that! But does it adhere to evidence-informed practices? Transl Behav Med. 2011;1:523-9.

8. Conroy DE, Yang $\mathrm{CH}$, Maher JP. Behavior change techniques in top-ranked mobile apps for physical activity. Am J Prev Med. 2014;46:649-52.

9. Cowan LT, Van Wagenen SA, Brown BA, Hedin RJ, Seino-Stephan Y, Hall PC, West $\mathrm{JH}$. Apps of steel: Are exercise apps providing consumers with realistic expectations?: A content analysis of exercise apps for presence of behavior change theory. Health Educ Behav. 2013;40:133-9.

10. Derbyshire E, Dancey D. Smartphone medical applications for women's health: What is the evidence-base and feedback? Int J Telemed Appl. 2013; 2013:782074.

11. Direito A, Dale LP, Shields E, Dobson R, Whittaker R, Maddison R. Do physical activity and dietary smartphone applications incorporate evidencebased behaviour change techniques? BMC Public Health. 2014;14:646.

12. Hermawati S, Lawson G. Managing obesity through mobile phone applications: a state-of-the-art review from a user-centred design perspective. Pers Ubiquitous Comput. 2014;18:2003-23.

13. Lyons EJ, Lewis ZH, Mayrsohn BG, Rowland JL. Behavior change techniques implemented in electronic lifestyle activity monitors: A systematic content analysis. J Med Internet Res. 2014;16:e192.

14. Middelweerd A, Mollee JS, van der Wal CN, Brug J, te Velde SJ. Apps to promote physical activity among adults: a review and content analysis. Int J Behav Nutr Phys Act. 2014;11:9.

15. Millington B. Smartphone apps and the mobile privatization of health and fitness. Crit Stud Media Commun. 2014;31:479-93.

16. Pagoto S, Schneider K, Jojic M, DeBiasse M, Mann D. Evidence-based strategies in weight-loss mobile apps. Am J Prev Med. 2013:45:576-82.

17. Rusin M, Årsand E, Hartvigsen G. Functionalities and input methods for recording food intake: a systematic review. Int J Med Inf. 2013;82:653-64.

18. Schoffman DE, Turner-McGrievy G, Jones SJ, Wilcox S. Mobile apps for pediatric obesity prevention and treatment, healthy eating, and physical activity promotion: Just fun and games? Transl Behav Med. 2013;3:320-5.

19. Silva PA, Holden K, Nii A: Smartphones, smart seniors, but not-so-smart apps: A heuristic evaluation of fitness apps. In foundations of augmented cognition. Advancing human performance and decision-making through 
adaptive systems. Edited by Schmorrow DD, Fidopiastis CM. Springer International Publishing, Switzerland; 2014:347-358. [Lecture Notes in Computer Science]

20. Watkins I, Kules B, Yuan X, Xie B. Heuristic evaluation of healthy eating apps for older adults. J Consum Health Internet. 2014;18:105-27.

21. Wearing J, Nollen N, Befort C, Davis AM, Agemy CK. iPhone app adherence to expert-recommended guidelines for pediatric obesity prevention. Child Obes. 2014;10:132-44.

22. West JH, Hall PC, Hanson CL, Barnes MD, Giraud-Carrier C, Barrett J. There's an app for that: content analysis of paid health and fitness apps. J Med Internet Res. 2012;14:184-95.

23. West JH, Hall PC, Arredondo V, Berrett B, Guerra B, Farrell J. Health behavior theories in diet apps. J Consum Health Internet. 2013;17:10-24.

24. Garcia-Gomez JM, de la Torre-Diez I, Vicente J, Robles M, Lopez-Coronado M, Rodrigues JJ. Analysis of mobile health applications for a broad spectrum of consumers: a user experience approach. Health Informatics J. 2014;20:74-84.

25. Washburn RA, Szabo AN, Lambourne K, Willis EA, Ptomey LT, Honas J, Herrmann SD, Donnelly JE. Does the method of weight loss effect longterm changes in weight, body composition or chronic disease risk factors in overweight or obese adults?A systematic review. PLoS One. 2014;9:e109849.

26. Jakicic JM, Marcus BH, Gallagher Kl, Napolitano M, Lang W. Effect of exercise duration and intensity on weight loss in overweight, sedentary women: a randomized trial. JAMA. 2003:290:1323-30.

27. Swift DL, Johannsen NM, Lavie CJ, Earnest CP, Church TS. The role of exercise and physical activity in weight loss and maintenance. Prog Cardiovasc Dis. 2014;56:441-7.

28. NICE, National Institute for Health and Clinical Excellence: Maintaining a Healthy Weight and Preventing Excess Weight Gain among Adults and Children | 1-Recommendations | Guidance and Guidelines | NICE. NICE public health guidance 7. NICE, National Institute for Health and Clinical Excellence; 2015[http://www.nice.org.uk/guidance/NG7/chapter/1recommendations. Accessed 9 Apr 2015-04-09; Archived by WebCite ${ }^{\oplus}$ at http://www.webcitation.org/6Xf2dRefW].

29. Bell K: Google play now has more apps than Apple's app store, report says. Mashable 2015 [http://mashable.com/2015/01/15/google-play-more-appsthan-ios. Accessed 16 Jan 2015; Archived by WebCite ${ }^{\oplus}$ at http://www. webcitation.org/6VenAx9TE].

30. Wallenstein A: How google play is closing the app gap with apple. Variety 2015 [http://variety.com/2015/digital/features/google-play-apple-itunes-app-store1201449326/. Accessed 9 Apr 2015; Archived by WebCite ${ }^{\circledast}$ at http://www. webcitation.org/6XfBOAiTj].

31. Gartner Says Smartphone Sales Surpassed One Billion Units in 2014 [http:// www.gartner.com/newsroom/id/2996817. Accessed: 2015-04-08. (Archived by WebCite ${ }^{\oplus}$ at http://www.webcitation.org/6Xdie1jav)] [http://www.gartner. com/newsroom/id/2996817. Accessed 8 Apr 2015; Archived by WebCite ${ }^{\oplus}$ at http://www.webcitation.org/6Xdie1jav]

32. Android and iOS Squeeze the Competition, Swelling to $96.3 \%$ of the Smartphone Operating System Market for Both 4Q14 and CY14, According to IDC [http://www.idc.com/getdoc.jsp?containerld=prUS25450615. Accessed: 2015-04-08. (Archived by WebCite ${ }^{\oplus}$ at http://www.webcitation. org/6XdikpM9S)] [http://www.idc.com/getdoc. jsp?containerld=prUS25450615. Accessed 8 Apr 2015; Archived by WebCite ${ }^{\oplus}$ at http://www.webcitation.org/6XdikpM9S]

33. Abraham C, Michie S. A taxonomy of behavior change techniques used in interventions. Health Psychol. 2008;27:379-87.

34. Michie S, Ashford S, Sniehotta FF, Dombrowski SU, Bishop A, French DP. A refined taxonomy of behaviour change techniques to help people change their physical activity and healthy eating behaviours: The CALO-RE taxonomy. Psychol Health. 2011;26:1479-98.

35. Michie S, Richardson M, Johnston M, Abraham C, Francis JJ, Hardeman W, Eccles MP, Cane J, Wood CE. The behavior change technique taxonomy (V1) of 93 hierarchically clustered techniques: building an international consensus for the reporting of behavior change interventions. Ann Behav Med. 2013:46:81-95.

36. Dombrowski SU, Sniehotta FF, Avenell A, Johnston M, MacLennan G, Araújo-Soares $\mathrm{V}$. Identifying active ingredients in complex behavioural interventions for obese adults with obesity-related co-morbidities or additional risk factors for co-morbidities: a systematic review. Health Psychol Rev. 2012;6:7-32.

37. Hartmann-Boyce J, Johns DJ, Jebb SA, Aveyard P, Behavioural Weight Management Review Group. Effect of behavioural techniques and delivery mode on effectiveness of weight management: systematic review, metaanalysis and meta-regression. Obes Rev. 2014;15:598-609.

38. Nielsen J: Usability inspection methods. In: Conference companion on Human factors in computing systems. ACM, New York, NY, USA; 1994:413414.

39. Nielsen J: Finding usability problems through heuristic evaluation. In: Proceedings of the SIGCHI conference on Human factors in computing systems. ACM, New York, NY, USA; 1992:373-380.

40. Stoyanov SR, Hides L, Kavanagh DJ, Zelenko O, Tjondronegoro D, Mani M. Mobile app rating scale: a new tool for assessing the quality of health mobile apps. JMIR MHealth UHealth. 2015;3:e27.

41. Xu W, Liu Y. mHealthApps: A repository and database of mobile health apps. JMIR MHealth UHealth. 2015;3:e28.

42. Garg R, Telang R: Inferring App Demand from Publicly Available Data. MIS Quarterly 2013:37(4):1253-1264.

43. Tang J, Abraham C, Greaves C, Yates T. Self-directed interventions to promote weight loss: A systematic review of reviews. J Med Internet Res. 2014;16:e58.

44. Gwet KL. Handbook of inter-rater reliability: the definitive guide to measuring the extent of agreement among multiple raters, 3rd edition. 3rd ed. Gaithersburg: Advanced Analytics, LLC; 2012.

45. Krippendorff K: Computing Krippendorff's alpha reliability. Dep Pap ASC 2011:43 [http://repository.upenn.edu/asc_papers/43]. Accessed 9 Apr 2015.

46. Girardello A, Michahelles F. AppAware: Which mobile applications are hot? In: Proceedings of the 12th international conference on human computer interaction with mobile devices and services. New York, NY, USA: ACM; 2010. p. 431-4. MobileHCl'10.

47. Carver CS, Scheier MF. Control theory: A useful conceptual framework for personality-social, clinical, and health psychology. Psychol Bull. 1982;92:111.

\section{Submit your next manuscript to BioMed Central and we will help you at every step:}

- We accept pre-submission inquiries

- Our selector tool helps you to find the most relevant journal

- We provide round the clock customer support

- Convenient online submission

- Thorough peer review

- Inclusion in PubMed and all major indexing services

- Maximum visibility for your research

Submit your manuscript at www.biomedcentral.com/submit 